\section{Seroprevalencia de virus hepatitis E en donantes de sangre en un hospital universitario en Chile}

\author{
Natalia Covarrubias', Paulina Naveas², Julio Miranda', \\ Carmen Hurtado', Daniela B. Vera', Milton Larrondo', \\ Javier Brahm' y Mauricio Venegas'
}

\section{Hepatitis E virus seroprevalence in blood donors in a university hospital in Chile}

In Chile, there are few studies about seroprevalence of $\operatorname{IgG}$ antibodies against hepatitis E virus (HEV) in blood banks, between 4 and $8 \%$. The development of new techniques with greater sensitivity and specificity, account for an increase in the seroprevalence of HEV in various countries, the current status in Chile being unknown. In the present study, we determined the seroprevalence of anti-HEV IgG in blood donors of the Clinical Hospital University of Chile, with last generation ELISA techniques. Out of a total of 186 samples, collected in 2014, $56(30.1 \%)$ were positive, without gender differences, but with a significant increase with age $(\mathrm{p}<0.001)$. These results show an increase in the seroprevalence of HEV in blood donors performed with immunoassays of greater sensitivity.

Key words: Hepatitis E Virus; seroprevalence; blood donors.

Palabras clave: Virus hepatitis E; seroprevalencia; donantes de sangre.

\section{Introducción}

E 1 virus hepatitis $\mathrm{E}$ (VHE) es una de las principales causas de hepatitis aguda en el mundo, estimándose que anualmente ocurren 20 millones de infecciones; de las cuales, 3 millones corresponden a casos sintomáticos, con alrededor de 55.000 muertes $^{1,2}$.

El VHE es un virus ARN sin envoltura, perteneciente al género Orthohepevirus de la familia Hepeviridae ${ }^{3}$. Se ha descrito un serotipo y se han identificado cuatro genotipos capaces de infectar a seres humanos. Los genotipos 1 y 2 han sido restringidos a seres humanos, mientras que los genotipos 3 y 4 , principalmente a animales ${ }^{4}$. El curso de la infección por VHE es generalmente benigno, excepto en mujeres embarazadas y pacientes con enfermedad hepática crónica, donde se han descrito casos de hepatitis fulminante. Además, se ha reportado la infección crónica por VHE genotipo 3, principalmente en pacientes inmunocomprometidos ${ }^{1}$.

Hospital Clínico Universidad de Chile. Santiago, Chile.

'Sección de Gastroenterología.

2Programa de Formación de especialista en Laboratorio Clínico, Universidad de Chile.

Banco de Sangre.

Conflictos de interés: no existen.

Fuente de Financiamiento: Oficina de Apoyo a la Investigación Clínica del Hospital Clínico Universidad de Chile.

Recibido: 8 de noviembre de 2017 / Aceptado: 3 de mayo de 2018

Correspondencia a:

Natalia Covarrubias Ruz

ncovarrubias@hcuch.cl
Actualmente, los estudios de seroprevalencia del VHE son difíciles de interpretar, presentando amplias variaciones según la población estudiada. En Europa se han comunicado tasas de seroprevalencia entre 0,6 y $52,5 \%$. Esta alta variabilidad ha sido atribuida a las características de la población, a la ubicación geográfica y principalmente a la sensibilidad de los inmunoensayos utilizados ${ }^{1}$. Por otra parte, en Latinoamérica, la mayoría de los estudios muestran tasas de seroprevalencia en población rural y urbana que varían entre 1 y $20 \%{ }^{5}$. Se ha planteado que el uso de nuevos ensayos de IgG anti-VHE más sensibles, ha llevado a un aumento de 3 a 4 veces las estimaciones de seroprevalencia. Esta diferencia debe ser considerada al interpretar los datos que actualmente están disponibles ${ }^{3,4,6}$.

En Chile, estudios realizados en donantes de sangre en los años 1996 y 1997 reportaron seroprevalencias de 4\% en Santiago y 8\% en Valdivia, Osorno y Puerto Montt; respectivamente. Cabe destacar que ambos estudios fueron realizados utilizando los ensayos de "primera generación", que actualmente son cuestionados por su baja sensibilidad ${ }^{1,7,8}$. Un estudio realizado por nuestro grupo en el año 2015, en muestras de pacientes con hepatitis de etiología no precisada, encontró un aumento de la seroprevalencia de 7,3 a 32,6\% al re-analizar las muestras utilizando un nuevo ensayo ${ }^{9}$. Debido a que este incremento significativo se explica únicamente en el uso de un ensayo con mejor rendimiento analítico, es razonable plantear la necesidad de actualizar los datos existentes en los bancos de sangre en nuestro país.

El objetivo de este estudio fue determinar la seroprevalencia de VHE en donantes de sangre de la Región Metropolitana de Chile utilizando un ensayo con un mejor rendimiento analítico.

\section{Método}

Se evaluaron 186 muestras de donantes de sangre del Hospital Clínico Universidad de Chile (HCUCH), recolectadas entre marzo y abril del año 2014. Las muestras se encontraban almacenadas a $-20^{\circ} \mathrm{C}$. El estudio fue aprobado por el comité de ética local.

El análisis de anti-VHE se realizó mediante ELISA AccuDiag ${ }^{\mathrm{TM}} \mathrm{HEV}-$ IgG (Diagnostic Automation, E. U. A.), el cual reporta una sensibilidad y especificidad de 99,8\%. Las muestras se procesaron de acuerdo con las instrucciones del fabricante. La asociación entre las variables del estudio y la seropositividad de IgG anti-VHE se estimó mediante la prueba de chi cuadrado. Un $p<0,05$ fue considerado estadísticamente significativo. Los análisis estadísticos se realizaron utilizando el programa computacional SPSS, versión 21.0.

\section{Resultados}

De los 186 donantes de sangre estudiados, 80 (43\%) eran mujeres y 106 (57\%) hombres. La edad promedio fue 37 años (rango, 18-68 años). Se encontraron $56(30,1 \%)$ muestras positivas para IgG anti-VHE. La seroprevalencia aumentó significativamente con la edad, registrando tasas más bajas en sujetos entre 18 y 29 años $(17,2 \%)$, en comparación con los grupos 30-39 años, 40-49 años y $\geq 50$ años (21, 42,5 y 46,6\%; respectivamente, $\mathrm{p}<0,001$ ) (Figura 1). No hubo diferencias significativas en la seropositividad IgG anti-VHE entre hombres y mujeres (31,1 y 28\%; respectivamente). 


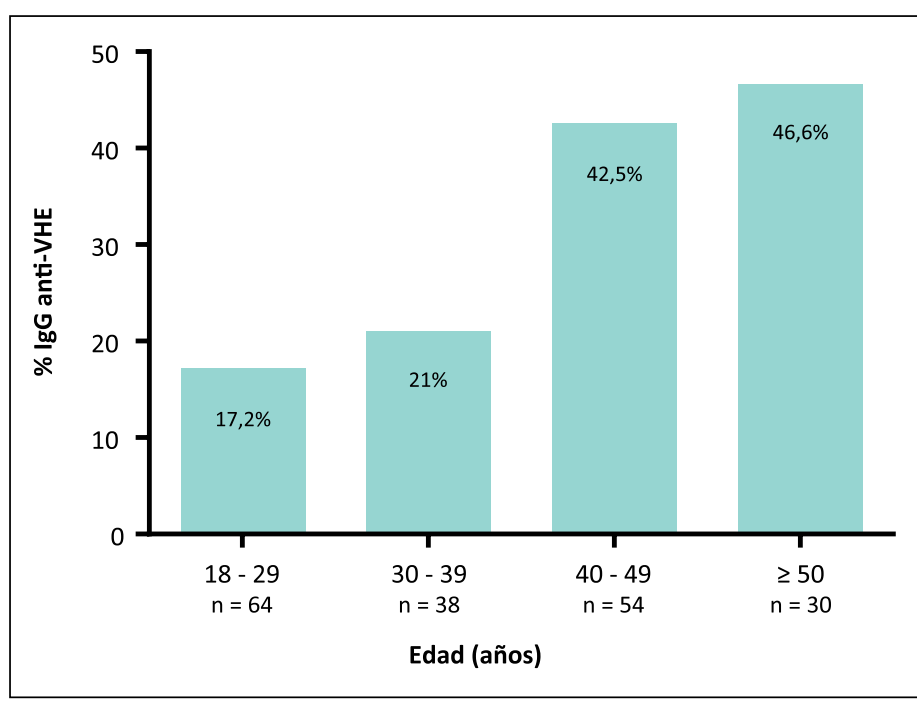

Figura 1. Prevalencia de IgG anti-VHE por grupo de edad en donantes de sangre en Hospital Clínico Universidad de Chile.

\section{Discusión}

Los resultados obtenidos muestran un incremento significativo en la seroprevalencia de VHE en donantes de sangre, respecto a estudios previos realizados en Chile en los años 1996 y 1997. Probablemente la razón más importante de este aumento es que dichos estudios utilizaron ensayos de IgG anti-VHE de "primera generación", los cuales subestimaron la seroprevalencia debido a su baja sensibilidad ${ }^{1,4,6-8}$. Es así como nuevos ensayos con mayor sensibilidad son capaces de detectar una mayor prevalencia de VHE, lo cual ha sido referido en diversas publicaciones ${ }^{1,3,6,9}$. Cabe destacar que dicho aumento no estaría asociado a una falta de especificidad del ensayo. Esto se ha descrito en población pediátrica y adulta con tasas de seroprevalencia muy bajas cuando se han utilizado ensayos con alta sensibilidad, descartando una posible sobre-estimación ${ }^{1,2,6}$.

De todas maneras, no podemos descartar que parte del aumento en la seroprevalencia de IgG anti-VHE encontrada, no se relacione únicamente con la sensibilidad del ensayo, sino que también corresponda a un incremento real. Un estudio realizado el año 2007 en muestras de suero de un grupo de cerdos destinados al consumo humano, en la zona central de nuestro país, encontró 7\% de IgG anti-VHE, lo cual demuestra que existe un reservorio de VHE porcino. Lo anterior, sumado a que el consumo per cápita de carne de cerdo en Chile se ha triplicado en los últimos 30 años, sugiere que los cambios en los hábitos alimenticios y las prácticas culinarias de nuestra población, podrían haber contribuido al aumento de seroprevalencia encontrado ${ }^{10}$.

Es interesante mencionar que al igual que nuestros resultados, diversos estudios reportan una elevada exposición de la población a VHE, lo que contrasta con el número relativamente bajo de casos sintomáticos des$\operatorname{critos}^{2,4}$. En nuestro país, un estudio de seroprevalencia del año 2015, en pacientes con solicitud médica de anticuerpos anti-VHE IgG que acudieron al HCUCH, encontraron resultados similares a los obtenidos en este estudio realizado en donantes de sangre (32,6 vs 30,1\% $)^{9}$. Esto sugiere que la mayoría de las infecciones en nuestro medio serían asintomáticas y no requieren atención médica. En Chile, se desconoce el genotipo predominante, lo cual sería importante para la interpretación de los resultados obtenidos, ya que se ha descrito que entre $67-98 \%$ de las infecciones con VHE genotipos 3 y 4 son asintomáticas ${ }^{11}$.

Con respecto al aumento significativo de la seroprevalencia con la edad, este resultado contrasta con el estudio realizado en nuestro país en 1997, que no mostró tal correlación ${ }^{8}$. Actualmente, existe evidencia internacional que apoya la correlación de la edad con mayores tasas de seroprevalencia de IgG anti-VHE ${ }^{1,3}$. Esto se explica fundamentalmente porque los ensayos de "primera generación" no son capaces de detectar títulos bajos de anticuerpos, teniendo muy baja sensibilidad para detectar infecciones previas. La asociación encontrada por nuestro grupo probablemente refleja la exposición al VHE acumulada en el tiempo de vida y/o a la persistencia de anticuerpos, condiciones que parecen ser similares en hombres y mujeres. Diversos estudios demuestran que los títulos de $\mathrm{IgG}$ anti-VHE se mantienen altos hasta 4,5 años después de la infección aguda, detectándose incluso 14 años posterior a la infección ${ }^{12}$.

Es esencial que los estudios de seroprevalencia se realicen con ensayos IgG anti-VHE sensibles, que nos permitan comparar los resultados obtenidos en las distintas poblaciones, sin la influencia y variabilidad del ensayo utilizado $^{3}$. Adicionalmente, debido a la alta prevalencia encontrada, podría ser necesario complementar el estudio con técnicas de biología molecular y de este modo evaluar el potencial riesgo de transmisión por vía sanguínea en nuestro medio.

\section{Resumen}

En Chile, existen escasos estudios de seroprevalencia de anticuerpos IgG anti virus hepatitis E (VHE) en bancos de sangre, entre 4 y $8 \%$. El desarrollo de nuevas técnicas con mayor sensibilidad y especificidad, dan cuenta de un aumento de la seroprevalencia de VHE en diversos países, siendo desconocido el estado actual en Chile. En el presente estudio, determinamos la seroprevalencia de IgG anti VHE en donantes de sangre del Hospital Clínico Universidad de Chile, con técnicas de ELISA de última generación. De un total de 186 muestras, recolectadas el año 2014, $56(30,1 \%)$ resultaron positivas, sin diferencias de género, pero con un incremento significativo con la edad $(\mathrm{p}<0,001)$. Estos resultados muestran un aumento en la seroprevalencia de VHE en donantes de sangre realizados con inmunoensayos de mayor sensibilidad.

\section{Referencias bibliográficas}

1.- Hartl J, Otto B, Madden R G, Webb G, Woolson K L, Kriston L, et al. Hepatitis E seroprevalence in Europe: a meta-analysis. Viruses 2016; 8: 211-24. doi: 10.3390/v8080211.

2.- Ankcorn M J, Tedder R S. Hepatitis E: the current state of play. Transfus Med 2017; 27: 84-95. doi: 10.1111/tme. 12405.

3.- Kamar N, Dalton H R, Abravanel F, Izopet J. Hepatitis E virus infection. Clin Microbiol Rev 2014; 27: 116-38. doi: 10.1128/CMR.00057-13.

4.- Arends J E, Ghisetti V, Irving W, Dalton H R, Izopet J, Hoepelman A I, et al. Hepatitis E: an emerging infection in high income countries. J Clin Virol 2014; 59: 81-8. doi: 10.1016/j.jcv.2013.11.013.

5.- Echevarría J M, González J E, Lewis-Ximenez L L, Dos Santos D R, Munné M S, Pinto M A, et al. Hepatitis E virus infection in Latin America: a review. J Med Virol 2013; 85: 1037-45. doi: 10.1002/jmv.23526. 
6.- Wenzel J J, Preiss J, Schemmerer M, Huber B, Jilg W. Test performance characteristics of anti-HEV IgG assays strongly influence hepatitis E seroprevalence estimates. J Infect Dis 2013; 207: 497-500. doi: 10.1093/ infdis/jis688.

7.- Brahm J, Hurtado C, Moraga M, Gil LC, Velasco M, Alegría S, et al. Hepatitis E virus infection in Chile: preliminary report. Rev Med Chile 1996; 124: 947-9.

8.- Ibarra H, Riedemann S, Reinhardt G, Frieck P, Siegel F, Toledo C, et al. Prevalence of hepatitis E virus antibody in blood donors and other population groups in Southern Chile. Rev Med Chile 1997; 125: 275-8.

9.- Covarrubias N, Hurtado C, Díaz A, Mezzano G, Brahm J, Venegas M.
Re-evaluación de seroprevalencia de virus hepatitis E. Rev Chilena Infectol 2015; 32: 482-4. doi: 10.4067/S0716-10182015000500020.

10.- Ibarra V H, Riedemann G S, Reinhardt V G, Calvo A M. Presencia de antiVHE en un estudio de cohorte de porcinos ¿reservorio animal de hepatitis $\mathrm{E}$ en Chile?. Rev Med Chile 2007; 135: 997-1001.

11.- Cleland A, Smith L, Crossan C, Blatchford O, Dalton H R, Scobie L, et al. Hepatitis E virus in Scottish blood donors. Vox Sang 2013; 105: 283-9. doi: 10.1111/vox.12056.

12.- Park H K, Jeong S H, Kim J W, Woo B H, Lee D H, Kim H Y, et al. Seroprevalence of anti-hepatitis E virus (HEV) in a Korean population: comparison of two commercial anti-HEV assays. BMC Infect Dis 2012; 12: 142. 